\title{
ON THE INTERACTION OF A MICROWAVE EXCITED OXYGEN PLASMA WITH A JET OF PRECURSOR MATERIAL FOR DEPOSITION APPLICATIONS
}

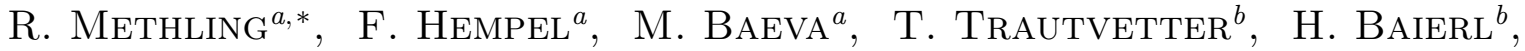 \\ R. FOEST $^{a}$ \\ ${ }^{a}$ Leibniz Institute for Plasma Science and Technology (INP), Felix-Hausdorff-Str. 2, 17489 Greifswald, Germany \\ ${ }^{b}$ Leibniz Institute of Photonic Technology (IPHT), Albert-Einstein-Str. 9, 07745 Jena, Germany \\ * methling@inp-greifswald.de
}

\begin{abstract}
A plasma source based on a microwave discharge at atmospheric pressure is used to produce an oxygen plasma torch. A liquid precursor material is evaporated and injected into the torch through a nozzle, causing oxidization and deposition of silica at a nearby quartz substrate. The temperature generated inside the plasma source and in the plume, in the region of treatment, and at the substrate surface are key parameters, which are needed for process description and optimization of plasma-chemical reactions. Optical emission spectroscopy and thermography were applied to observe and characterize the jet behavior and composition. The experimental results are compared with self-consistent modeling.
\end{abstract}

Keywords: microwave plasma, plasma jet, optical emission spectroscopy, thermography.

\section{Introduction}

High performance glasses based on doped silica are essential parts of modern fiber based laser systems [1]. They provide highest precision and flexibility in system design combined with high power output and excellent beam quality. In case of pulsed operation an excellent material homogeneity is required due to high pulse peak powers.

Existing fiber preform fabrication technologies such as modified chemical vapor deposition (MCVD) gas phase or solution doping, reactive powder sintering technology (REPUSIL) or the common melt process face limitations regarding the feasibility of achievable geometrical as well as chemical dopant specifications and thus the optical performance of the resulting core material. For instance, constraints in terms of diameter of active core, homogeneity of dopant distribution within the core as well as the practicable laser active dopant concentration affect the resulting optical performance of the fabricated fiber, especially its spectral attenuation and photo darkening behavior. Furthermore, laser active dopants increase the refractive index so that a co-doping with a dopant decreasing the refractive index is required. The interaction of the two dopants usually confines, for thermodynamic reasons, the accessible core specification in terms of dopant concentration profiles, maximum dopant concentrations, and core diameter.

To overcome such thermodynamic limitations, a novel approach based on atmospheric-pressure microwave (MW) plasma has been elaborated. Previous research has demonstrated that plasma based technology at atmospheric pressure is a valuable tool for the treatment of specialty glasses and optical fibers. The use of a plasma system at atmospheric pressure offers a wide range of advantages. Atmospheric pressure plasmas are generally characterized by a high density of active species resulting in higher reaction rates which in turn are capable of significantly increasing deposition rates and efficiencies compared to low pressure plasmas. Microwave plasmas offer an even higher intrinsic electron, ion and radical density thus enhancing deposition efficiencies in comparison to other plasma sources. Furthermore, electrode-less systems such as microwave and RF plasmas circumvent possible contamination sources as encountered with e.g. DC/AC plasma jets. However, the coupling efficiency of RF energy into the plasma is considered critical. Values as low as $40 \%$ to $50 \%$ are reported, dropping even more significantly with high power input above $100 \mathrm{~kW}$. Instead, the coupling efficiency of the incoming microwave power into the plasma can reach nearly $100 \%$ provided that the wave guide setup is configured correctly. The plasma-based approach could offer an outstanding advantage in terms of plasma-induced kinetic control of the deposition process. It thus allows for co-doping beyond thermodynamically determined chemical equilibrium, and for overcoming diffusion limitations by the processes controlled by convection. This feature results in an overall improved outstanding homogeneity of the doped core material.

In this paper, the setup of a microwave plasma system at atmospheric pressure for the deposition of $\mathrm{SiO}_{2}$ is described. Optical emission spectroscopy and thermography were applied to observe and characterize the jet behavior and composition as well as gas and target temperature. The experimental results are compared with self-consistent modeling. 


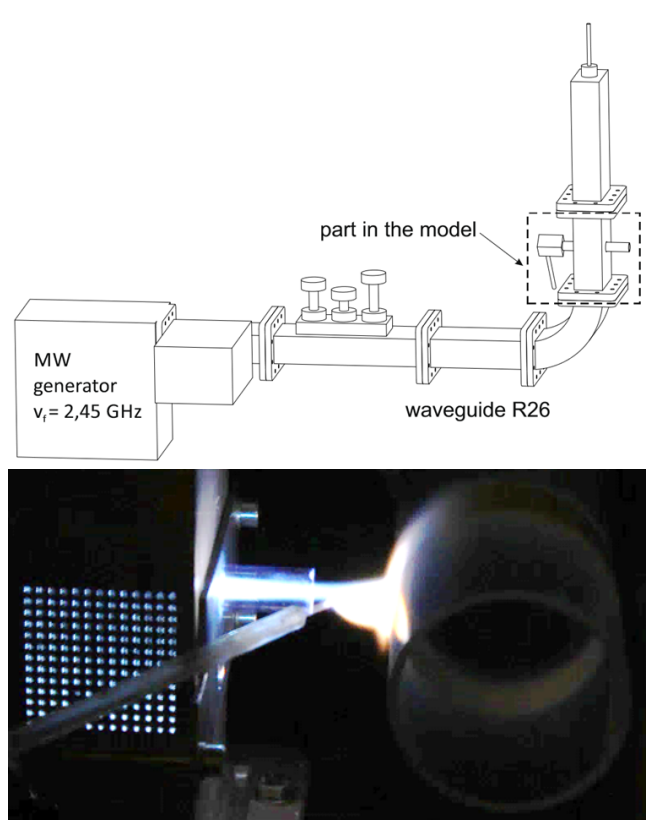

Figure 1. Top: Schematic view of the plasma source. The plasma torch is produced by excitation of the gas flow through the quartz tube traversing the waveguide. Bottom: Photograph of the plasma torch, precursor nozzle, and rotating substrate.

\section{Experimental Setup}

A microwave plasma torch operated at atmospheric pressure at a frequency of $2.45 \mathrm{GHz}$ was used as shown in the upper part of Figure 1. The plasma source consisted of a standard wave-guide R26 and a quartz tube of $12 \mathrm{~mm}$ inner diameter traversing at the position of maximum electric field of the fundamental mode TE 10. Mixtures of argon and oxygen, including the pure gases, were employed as working gas. The plasma jet was directed towards a rotating substrate as shown in the lower part of Figure 1. For the generation of silica, the precursor octamethylcyclotetrasiloxane (OMCTS) was either vaporized or nebulized by means of ultrasonic impact. With some Ar flow, the precursor was fed through an additional nozzle placed outside the plasma source in an angle of about $45^{\circ}$ towards the plasma torch. As a substrate a big quartz tube rotating perpendicular to the plasma (as shown in the photograph) was used.

Several techniques were applied for the optical analysis. The general behavior of the plasma with focus on the interaction region of the two jets and the deposition area was recorded by a high-speed video camera.

A $0.5 \mathrm{~m}$ imaging spectrometer (SP-2556, Roper Acton) equipped with an ICCD camera (PI-MAX4:1024i$\mathrm{RB}$, Princeton Instruments) was used to record the plasma emission along the MW jet, including positions inside the quartz tube as well as in the plasma plume. Therefore, the radiation was focused by a quartz lens onto the entrance of an UV-VIS fiber which was coupled to the spectrometer. While overview spectra as shown in the upper part of Figure 2 were taken with a grating of 50 lines per $\mathrm{mm}$, higher spectral resolu-
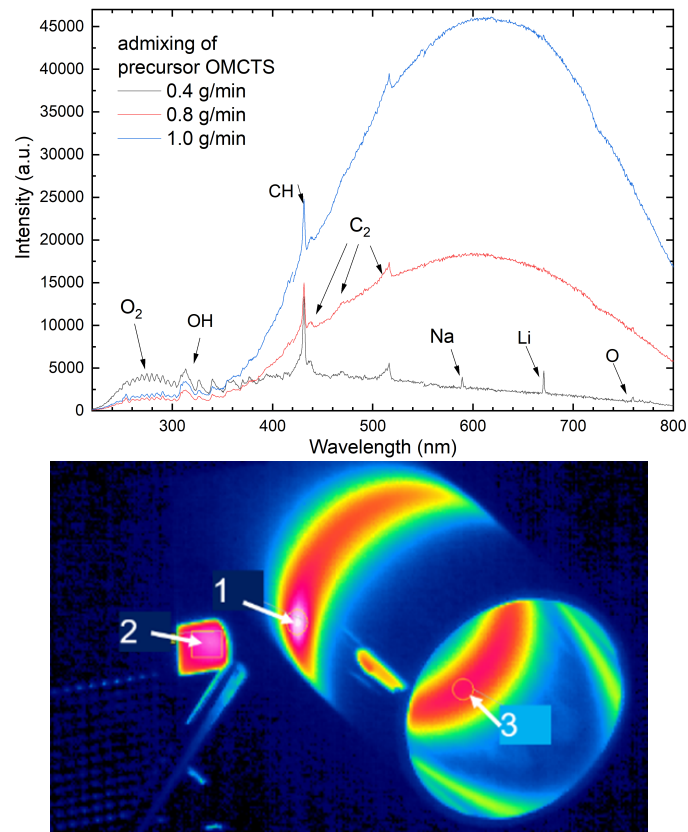

Figure 2. Top: Overview spectrum of oxygen plasma torch with different admixtures of OMCTS precursor. Bottom: Thermography image.

tion ( $\sim 0.03 \mathrm{~nm}, 2400 \mathrm{l} / \mathrm{mm}$ grating) was used for the analysis of molecular band structures.

Surface temperatures were determined by means of a thermographic camera (VarioCAM, InfraTec) with an effective resolution of $640 \times 480$ pixels. The measurement was set up in the temperature range $450-1500^{\circ} \mathrm{C}$ tube serving as deposition target. Time-averaged values were obtained for three positions: (1) the spot of deposition, (2) the end of the plasma torch quartz tube and (3) at the position of the local temperature maximum inside the substrate (lower part of Figure 2). The sensitivity of the measurement allowed detecting even small variations of few $\mathrm{mm}$ in the distance of operation.

The target was typically placed at a varying distance $L_{\text {tar }}$ from the wave guide. The position of spectroscopic investigation $x$ was related to the end of quartz tube $L_{\text {out }}$, i.e. the maximal value is at the position of the target $x=L_{\text {tar }}-L_{\text {out }}$.

\section{Self-Consistent Model}

The determination of the plasma parameters in the region of plasma-precursor interaction was of primary importance for the technological application. On the one hand, the plasma parameters in the plume depended on the conditions inside the plasma source. On the other hand, the source was hardly accessible for diagnostics. Therefore, complementary studies by modeling and experiments were carried out.

A self-consistent model was developed to describe the MW plasma source employing the plasma, electromagnetic and hydrodynamic equations[2]. It was realized on the computational platform COMSOL Multiphysics in two- and three-dimensional geometry[3]. 

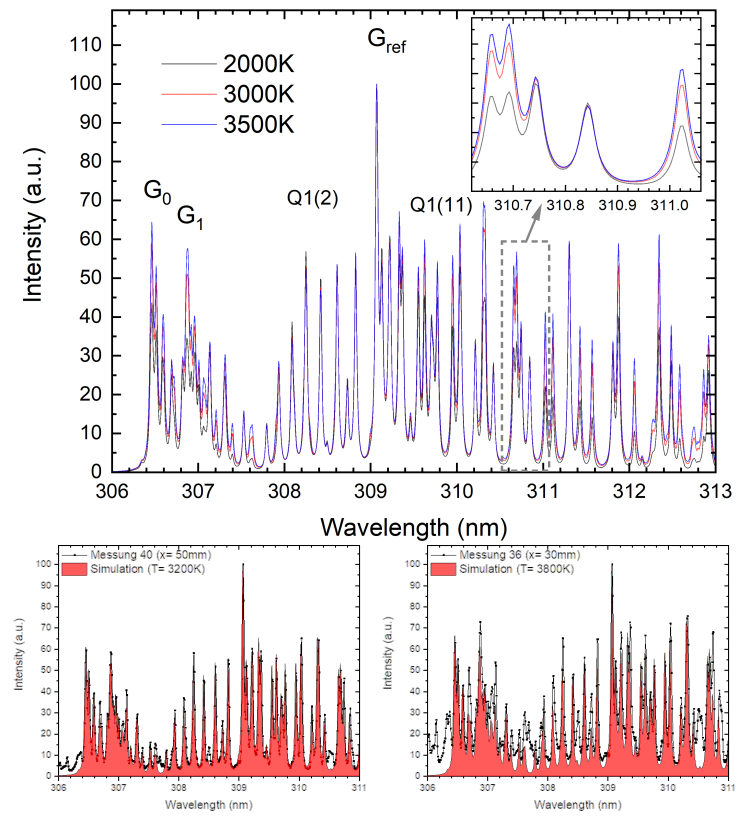

Figure 3. $O H$ band emission. Top: Calculated spectra for $2000 \mathrm{~K}, 3000 \mathrm{~K}$, and $3500 \mathrm{~K}$. Bottom: Comparison of recorded and calculated spectra with gas temperatures of about $3200 \mathrm{~K}$ (left) and $3800 \mathrm{~K}$ (right).

The microwave entered the wave guide, propagated along it and was reflected on the short end to build a standing wave. The gas was fed into a quartz tube. The tube position was adjusted to match the maximum of the electric field in the wave guide to ignite the plasma. The model equations described in a selfconsistent manner the gas flow, the plasma kinetics, the heat transfer, and the MW field in the waveguide. It was based on a hydrodynamic approach. The Navier-Stokes equations for conservation of mass and momentum, the MW field equation, and the equation for conservation species, energy of electrons and heavy particles were considered. The model avoided the assumption of quasi-neutrality and solved the Poisson equation to obtain the electrostatic potential. The results obtained in argon as a working gas[2] showed that for temperatures over $\sim 1350 \mathrm{~K}$ in the plasma, the atomic ion was dominant. The molecular ion played a role in the cold region near the tube wall. Since most of the plasma flow in the source was hot, the present studies were performed in the frame of the one-ion plasma chemistry.

\section{Gas Temperature Determination}

For the evaluation of gas temperature, the rotational temperature of the $\mathrm{OH}(\mathrm{A}-\mathrm{X})$ band with maximum intensity near $309 \mathrm{~nm}$ was identified by comparing recorded and calculated spectra. The calculations were performed using the simulation software Lifbase [4]. A small amount of $\mathrm{OH}$, which was present from water, e.g. due to diffusion from the surrounding air into the plasma plume zone, allowed to determine the gas temperature in the most cases. For $\mathrm{O}_{2}$-containing gas mixtures, an overlapping of the

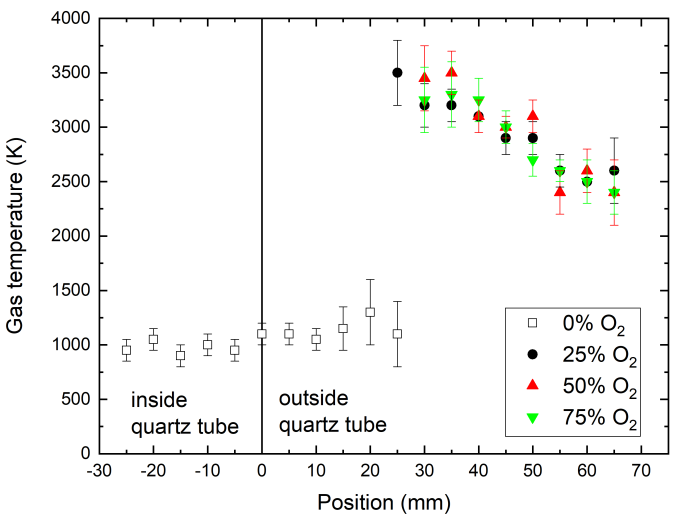

Figure 4. Gas temperatures determined according to Figure 3 for different $\mathrm{Ar} / \mathrm{O}_{2}$ mixtures.

$\mathrm{OH}(\mathrm{A}-\mathrm{X})$ bands with Schumann-Runge bands of $\mathrm{O}_{2}$ (about 200-450 nm with maximum intensity around $350 \mathrm{~nm}$ ) occurred, mainly for positions near to the plasma source. Thus, the $\mathrm{OH}$ band structures were accessible only downstream in the plume and no temperature determinations from spectroscopy were possible at the tube end and at the place of the precursor feed. A simulation of the spectral emission pattern for three gas temperatures is shown in the upper part of Figure 3. In the lower part of the figure, two examples of comparison of measured and calculated $\mathrm{OH}$-spectra are plotted for a distance of $-5 \mathrm{~mm}$ from near the tube end in pure argon.

\section{Results and Discussion}

The gas temperature at different $x$ positions along the MW torch was determined from $\mathrm{OH}$ spectra and compared with values extracted from simulation with the $2 \mathrm{D}$ planar model. Four $\mathrm{Ar} / \mathrm{O}_{2}$ gas mixtures emitted into a substrate-free space were investigated at an incoming $\mathrm{MW}$ power of $1.0 \mathrm{~kW}$, a gas inflow rate of $18 \mathrm{slm}$, and a tube length $\mathrm{L}_{\text {out }}=38 \mathrm{~mm}$. The results are summarized in Figure 4. With pure argon (0\% oxygen admixture) the temperature reached a maximum value of $\sim 1500 \mathrm{~K}$ close to the inner tube wall on the side of the incoming microwaves, decreasing outside the tube to temperatures far below $1000 \mathrm{~K}$. Interestingly, a simulated temperature decrease between the end of the wave guide $(-25 \mathrm{~mm})$ and the end of the quartz tube $(0 \mathrm{~mm})$ could not be found in the spectra. On the contrary, the gas temperatures deduced from emission spectroscopy showed a slight increase from about $1000 \mathrm{~K}$ inside the tube to $1200 \mathrm{~K}$ at a position $20 \mathrm{~mm}$ outside the tube. While the model predicted temperatures of about $600 \mathrm{~K}$ in the plume, the emission spectroscopy yielded values of $1000-1400 \mathrm{~K}$. The admixture of oxygen caused a significant change in the plasma and its properties. The gas temperature could only be determined from $\mathrm{OH}$ emission far away from the tube end, where emission from molecular oxygen was absent. It was found to be substantially higher for any admixture of oxygen than in pure argon. This result could be probably explained with the heat 
released in the reactive plasma due to dissociation of oxygen molecules. Temperatures for different $\mathrm{O}_{2}$ admixtures were similar within measurement accuracy. The temperature decreased nearly linear with the distance from about $3500 \mathrm{~K}(x=25 \mathrm{~mm})$ to $2400 \mathrm{~K}$ $(x=65 \mathrm{~mm})$. No plasma emission could be observed at distances $x>70 \mathrm{~mm}$.

Beside the above mentioned molecular bands of $\mathrm{OH}$ and $\mathrm{O}_{2}$, several other features appeared in the overview spectra in the cases with admixture of $\mathrm{Ar}$ and OMCTS precursor, cf. Figure 2. Molecules originating from the carbon-containing precursor, e.g. $\mathrm{CH}$ and $\mathrm{C}_{2}$, were detectable by their band heads. Furthermore, some atomic lines as $\mathrm{Na}$ and $\mathrm{Li}$ could be found in cases of high thermal load on the glass components of the setup while $\mathrm{O}$ lines only occurred for low amounts of precursor admixture. No ionic lines were observed, validating a low amount of charged particles and moderate gas temperatures.

Another specific feature of the spectra was the continuum emission above $\sim 500 \mathrm{~nm}$. Its intensity was very sensitive to the amount of OMCTS admixture, cf. overview spectra in Figure 2. Several sources are possible causing similar continuum radiation. (i) For a molecular radiation, a typical shape usually including local maxima or band heads would be expected. (ii) Recombination radiation is typically characterized by broad emission over several hundreds of nm with flat profiles without distinct lines or peaks. (iii) Thermal radiation has a spectral distribution according to the Planck function; in relevant wavelength range between VIS and NIR it is typically characterized by (nearly linear) increase towards longer wavelengths.

For advanced analysis, the influence of spectral sensitivity was eliminated by (relative) spectral calibration using a tungsten ribbon lamp. Figure 5 shows two calibrated spectra with a precursor flow of $2.0 \mathrm{slm}$ Ar plus 0.8 and $1.0 \mathrm{~g} / \mathrm{min}$ OMCTS. The spectral range between 550 and $800 \mathrm{~nm}$ was chosen only to reduce the influence from $\mathrm{CH}$ and $\mathrm{C}_{2}$ bands $(<550 \mathrm{~nm})$ and reduced sensitivity causes noise $(>800 \mathrm{~nm})$. The curves could be fitted very well to Planck functions obtaining temperatures of $3180 \mathrm{~K}$ for the lower $(0.8 \mathrm{~g} / \mathrm{min})$ and $2970 \mathrm{~K}$ for the higher amount of OMCTS $(1.0 \mathrm{~g} / \mathrm{min})$. It was double-checked that no thermal radiation from the target surface was detected by the OES system and the radiation must originate from the interaction area of the two jets (oxygen plasma and precursor) near to the target. In principle, a gas jet should not emit thermal radiation. However, an explanation might be found in micro-droplets surviving from the precursor injection. Here, NIR spectroscopy could help to reveal this phenomena.

Figure 6 shows the surface temperature of the quartz substrate obtained by thermographic measurements for various distances between the end of the discharge tube and the substrate. It should be noted that differently from the results shown above, the oxygen flow through the plasma source was kept constant

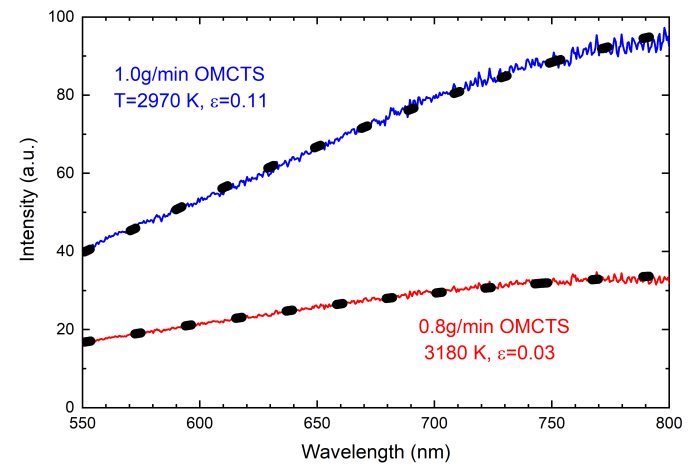

Figure 5. Part of spectra after relative intensity calibration $\left(1.0 \mathrm{~kW}, 10 \mathrm{slm} \mathrm{O}_{2}, L_{\text {out }}=25 \mathrm{~mm}, L_{\text {tar }}=45 \mathrm{~mm}\right.$, $x=15 \mathrm{~mm})$. The curves are fitted using Planck functions; temperatures of $\sim 3000 \mathrm{~K}$ are obtained.

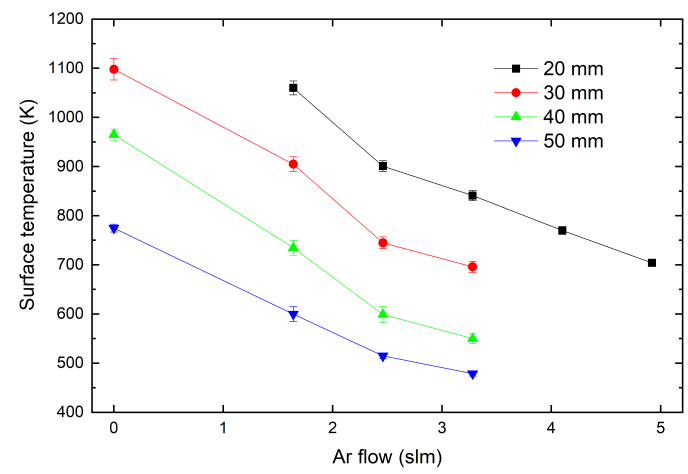

Figure 6. Target surface temperature depending on precursor gas flow for different target distances $x$.

and the argon was added via the precursor tube. Two well-pronounced effects were observed: On the one hand, the surface temperature was highest in pure oxygen and decreased with the increase of the Arconcentration due to gas cooling. On the other hand, the surface temperature decreased with increasing distance due to a reduced heating by the plasma jet.

\section{Acknowledgements}

The work was supported by grant SAW-2017-IPHT-1 of the Leibniz-Gemeinschaft. The authors acknowledge M. Stankov and J. Schäfer for helpful discussions.

\section{References}

[1] S. Addanki, I. S. Amiri, and P. Yupapin. Review of optical fibers-introduction and applications in fiber lasers. Results in Physics, 10:743-750, 2018. doi:10.1016/j.rinp. 2018.07.028.

[2] M. Baeva, F. Hempel, H. Baierl, T. Trautvetter, F. Foest, and D. Loffhagen. Two- and three-dimensional simulation analysis of microwave excited plasma for deposition applications: operation with argon at atmospheric pressure. J. Phys. D: Appl. Phys., 51:385202, 2018. doi:10.1088/1361-6463/aad537.

[3] COMSOL. COMSOL Multiphysics v. 5.3a. http://www . comsol. com.

[4] J. Luque and D. R. Crosley. SRI International Report MP 99-009, version 1.9. LIFBASE. 